\title{
Análise dialógica dos níveis, dimensões e indicadores de sustentabilidade
}

\author{
Alexandre André Feil ${ }^{2}$ e Dusan Schreiber ${ }^{2}$ \\ ${ }^{1}$ Universidade do Vale do Taquari (UNIVATES). Programa de Pós-Graduação em \\ Sistemas Ambientais Sustentáveis (PPSAS). Rua Avelino Talini, 171. Bairro \\ Universitário. Lajeado-RS, Brasil (CEP 95914-014). E-mail: \\ alexandre.feil1@gmail.com. \\ ${ }^{2}$ Universidade Feevale. Programa de Pós-Graduação em Qualidade Ambiental. Av. \\ Dr. Maurício Cardoso, 510. Hamburgo Velho. Novo Hamburgo-RS (CEP 93510- \\ 235).
}

Resumo. Este estudo objetiva realizar uma análise crítica dos níveis, das dimensões e dos indicadores de sustentabilidade. A metodologia utilizada é a qualitativa e bibliográfica. Os principais resultados revelam que os níveis de sustentabilidade vinculam-se à substituição do capital natural (ou não) por outros capitais existentes, e o nível mais adequado na prática compreenderia um meio termo entre o nível forte e fraco de sustentabilidade. As dimensões Triple Bottom Line (TBL) da sustentabilidade referemse à delimitação da área de abrangência, já a sustentabilidade centra-se na vitalidade dos sistemas, mantendo, protegendo e preservando-os. A análise do processo de elaboração e identificação dos indicadores de sustentabilidade revela que se deve observar as qualidades desejáveis e suas características, utilizando, de forma híbrida, a abordagem top-down e bottom-up. Portanto, salienta-se que os conceitos desses termos da sustentabilidade são escassos e dispersos na literatura. Dessa forma, este estudo compilou o conceito desses termos, corroborando com demais estudos centrados neste tema.

Palavras-chave: Triple bottom line; Top-down e bottom-up; Conceitos.

Abstract. Dialogical analysis of levels, dimensions and indicators of sustainability. This study aims at critical analyzing levels, dimensions and sustainability indicators. The methodology approach is qualitative and bibliographic research. The main results reveal that the levels of sustainability are connected to the substitution of natural capital (or not) for other existing capitals; the more adequate level, in practice, would be a middle ground between the strong and weak levels of sustainability. The dimensions of sustainability (triple bottom line) refer to the delimitation of the length or the area covered, whereas the sustainability encompassed by the triple bottom line centers in the vitality of the systems, keeping them, protecting them and preserving them. The analysis of the process of elaboration and
Recebido:

$12 / 04 / 2019$

Aceito:

$21 / 07 / 2019$

Disponível on line:

$22 / 07 / 2019$

Publicado:

$31 / 08 / 2019$

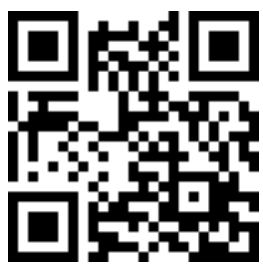

Acesso aberto

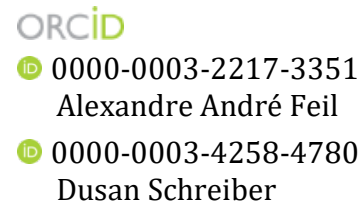


identification of the indicators of sustainability reveals that the desired qualities and its characteristics should be observed, using, in a hybrid way, the top-down and bottom-up approach. Therefore, we highlight that the concepts of these terms are scarce and scattered in the literature. This way, this study compiled the concepts of these terms, confirming with other studies about this subject.

Keywords: Triple bottom line; Top-down and bottom-up; Concepts.

\section{Introdução}

A sociedade depende, na dimensão material, dos recursos naturais para sua existência. Entretanto, as atividades humanas degradam e esgotam esses recursos e, assim, ameaçam o bemestar humano e, possivelmente, sua existência (Howard et al., 2014). Além disso, as reivindicações sobre as questões ambientais aumentaram ao longo dos últimos anos, exigindo uma postura mais proativa frente a sua preservação (Wickboldt et al., 2018). Dessa forma, o conceito de sustentabilidade e desenvolvimento sustentável emergiu para solucionar essas inquietações globais.

0 conceito de desenvolvimento sustentável mais citado e conhecido globalmente é definido como o "[...] desenvolvimento que satisfaz as necessidades do presente sem comprometer a capacidade das gerações futuras satisfazerem as suas próprias necessidades" (WCED, 1987). A sustentabilidade e o desenvolvimento sustentável não são sinônimos, apesar de sua similaridade. Sendo assim, neste estudo utiliza-se a ideia de sustentabilidade.

A sustentabilidade abrange três tipos de interesses simultâneos e em equidade: o ambiental, o econômico e o social, ou seja, Triple Bottom Line (TBL) (Horbach, 2005). A definição da sustentabilidade consiste na representação da alteração da propriedade do sistema; essa alteração vincula-se a sua qualidade (Bell e Morse, 2008).
A sustentabilidade é monitorada com o auxílio de indicadores (Dahl, 2012), os quais simplificam e quantificam os diversos fenômenos do sistema ambiental-humano, identificando, assim, tendências da qualidade desse sistema (Sartori et al., 2014). Entretanto, indicadores de sustentabilidade harmonizados e definidos com uniformidade de forma global estão em falta (Gaurav et al., 2011). Para Agol et al. (2014), os indicadores tradicionais não podem ser confundidos com os indicadores de sustentabilidade, por não dar conta de evidenciar as referidas alterações.

Os indicadores de sustentabilidade centram-se em oferecer informações confiáveis sobre o nível de sustentabilidade de um sistema local, nacional ou global (Singh et al., 2012). Destarte, este estudo objetiva realizar uma análise dos níveis, das dimensões e dos indicadores de sustentabilidade, para melhorar o entendimento e a clareza dessas questões essenciais na mensuração da sustentabilidade.

A justificativa deste estudo vincula-se na necessidade de uma profunda investigação sobre níveis, dimensões e indicadores de sustentabilidade, auxiliando assim no avanço na direção da sustentabilidade (Romero e Linares, 2014). A literatura aponta a urgência em encontrar indicadores eficazes para a sustentabilidade, pois os já existentes mensuram, principalmente, tendências insustentáveis no processo de gestão, mas ficam aquém de definir e assegurar a sustentabilidade (Dahl, 2012). Rocha et 
al. (2017) enfatizam que há uma necessidade de aprofundamento de pesquisas e discussões sobre a sustentabilidade. Sendo assim, contribui-se com o entendimento dos níveis, dimensões e dos indicadores de sustentabilidade, pois antes de iniciar a identificação e/ou definição de indicadores, é necessário o entendimento desses diversos conceitos relacionados.

\section{Metodologia}

O tipo de pesquisa, neste estudo, quanto à abordagem do problema é a qualitativa, pois consiste na análise e interpretação de informações textuais, tais como conceitos e ideias, extraídos da literatura científica. Já quanto ao procedimento técnico, vincula-se à pesquisa bibliográfica, na qual as informações utilizadas referem-se a artigos e livros elaborados com método e rigor científico.

As bibliografias para base da pesquisa bibliográfica foram coletadas por meio da consulta aos sites dos periódicos da Science Direct e do Google Scholar. A coleta das bibliografias abrangeu apenas as referências com idioma em inglês, considerando publicações nacionais e estrangeiras. Salienta-se que não houve recorte temporal do período de publicação, pois se resgatou bibliografias desde $o$ surgimento dos conceitos até a atualidade.

Foram utilizadas palavras-chave para a identificação da bibliografia, na aba de pesquisa avançada nos periódicos da Science Direct e do Google Scholar. As pesquisas foram realizadas por assunto. Quanto aos níveis de sustentabilidade, as palavras-chave utilizadas foram: strong, very strong; weak; e very weak sustainability; dessa forma, localizou-se na Science Direct 101 referências, e no Google Scholar, 180; destas, após leitura dos títulos e dos abstracts, foram selecionadas apenas 21 bibliografias, que foram lidas e utilizadas na seção. Já quanto a dimensões de sustentabilidade ambiental, social e econômica, utilizaram-se as palavras-chave: dimensions, environmental sustainability, social sustainability, economic sustainability, concept, definition, no Science Direct, foram identificadas 180 referências; no Google Scholar, 142; após a leitura inicial dos títulos e dos abstracts, foram selecionadas 30 bibliografias, que compõem a seção. Para indicadores de sustentabilidade, foram utilizadas as palavras-chave: indicators of sustainability; sustainability metrics; concepts; qualities of indicators; disadvantages; critical; foram identificados no Science Direct 74 referências; no Google Scholar, 6; após leitura prévia dos títulos e dos abstracts, foram selecionadas 55 bibliografias.

Cabe ressaltar que os conteúdos das bibliografias pesquisadas também contribuíram entre as seções. Por exemplo, a seção indicadores de sustentabilidade também se valeu das referências da seção dimensão $e$ sustentabilidade. Sendo assim, a coleta total foi de 106 estudos e documentos científicos. 0 período de coleta desses estudos foi de agosto a novembro de 2015.

O tipo de análise empregado é a interpretativa que consiste na interpretação do conteúdo para alcançar o significado profundo da comunicação escrita, a comparação das ideias entre diferentes autores e uma análise crítica de posicionamento, que ocorreu de setembro a dezembro de 2016, e logo se realizou a elaboração das seções subsequentes. Sendo assim, destaca-se que este estudo não teve a pretensão de abranger na sua totalidade as bibliografias existentes sobre o tema supracitado, e sim coletar informações suficientes para satisfazer adequadamente o escopo central desta pesquisa. 


\section{Resultados e discussão}

\section{Classificação dos níveis de sustentabilidade}

A sustentabilidade, segundo Goodland e Daly (1996) e Ekins et al. (2003), pode ser classificada como muito fraca, fraca, forte (Blewitt, 2008) e muito forte. Esses níveis de sustentabilidade consideram os tipos de capital analisados (Blewitt, 2008): a) humano (educação e habilidades); b) social (relações sociais e redes); c) natural (recursos naturais e serviços e processos do ecossistema, tais como o ar, a água, a terra e os habitats) (Ekins et al., 2003); e d) manufaturado (produtos, tecnologias).

0 conceito de sustentabilidade fraca e muito fraca surgiu no estudo de Solow (1974), definido como paradigma da substituição perfeita, em função do crescimento econômico com base nos recursos naturais não renováveis utilizados na produção. A sustentabilidade muito fraca consiste na substituição completa do capital natural por outros capitais (Turner, 1993; Neumayer, 2003). Nesta sustentabilidade, a perda de capital natural não deve ser superior ao aumento de capital humano e manufaturado (Blewitt, 2008). Portanto, a sustentabilidade muito fraca ocorre quando os capitais manufaturado, humano e o social substituem perfeitamente os serviços prestados pelo capital natural. Sendo assim, o bem-estar não estaria condicionado a algum capital específico.

$\mathrm{Na}$ sustentabilidade fraca, o capital manufaturado substitui o capital natural, embora com exceções (Ayres, 2007). Neste nível, é permitida a diminuição da quantidade de capital natural, e este é substituído pelo aumento de outras formas de capital (Pisani, 2006). Porém, esta redução de capital não pode ser menor que a quantidade mínima de capital natural crítico necessária para assegurar as funções em longo prazo (Blewitt, 2008). Fiorino (2011) interpreta-a como o aumento do bem-estar econômico.
Entende-se que na sustentabilidade fraca não há diferença entre os tipos de capital (natural, manufaturado, social e humano) e o bem-estar gerado. Nessa lógica, neste nível, enquanto que os estoques de capital natural diminuem, estes são substituídos por outros; assim, o capital total continuará constante, e o nível de consumo atual pode continuar.

A sustentabilidade forte e muito forte originou-se de Daly (1992), e define-as a partir da ideia de que muitos serviços prestados pelo capital natural não podem ser substituídos, em nenhum nível, pelo capital manufaturado. Sendo assim, a sustentabilidade forte sofre uma limitação da substituição do capital natural pelo capital manufaturado, assumindo-se que alguns componentes ambientais possuem contribuição única para o bem-estar, e essas contribuições podem ser irreversivelmente perdidas com o tempo (Ayres, 2007; Garmendia et al., 2010).

$\mathrm{Na}$ sustentabilidade forte, segundo Pisani (2006), não pode haver um declínio do capital natural ao longo do tempo; a herança de capital das gerações futuras deve ser o mesmo das gerações anteriores. Bell e Morse (2008) destacam que no nível forte há pouca consideração dos aspectos financeiros e de custos para alcançar a sustentabilidade. $E$ a sustentabilidade muito forte não aceita substituição do capital natural por outros, o qual deve ser totalmente conservado (Turner, 1993).

Na sustentabilidade forte há uma limitação na substituição do capital natural frente a outros capitais (produzidos, sociais, entre outros), pois alguns componentes do capital natural não podem ser substituídos, por exemplo, a qualidade do ar que, ao ser destruída, compromete os interesses e o bem-estar das gerações futuras. Já a muito forte não admite a substituição do capital natural pelo manufaturado, social e humano, ou seja, ele deve ser preservado na íntegra (Fiorino, 2011).

Em suma, afirma-se que o capital natural é a chave de diferenciação dos 
níveis de sustentabilidade e seu foco centraliza-se no sistema natural, considerando ou não suas compensações em comparação com outros capitais (Figura 1). Assim, entende-se que o capital natural é essencial para a sobrevivência humana, pois fornece as funções básicas, como alimentos, água, ar respirável e um clima estável para a sobrevivência e o bem-estar. Esta percepção corrobora Lahtinen et al. (2014).

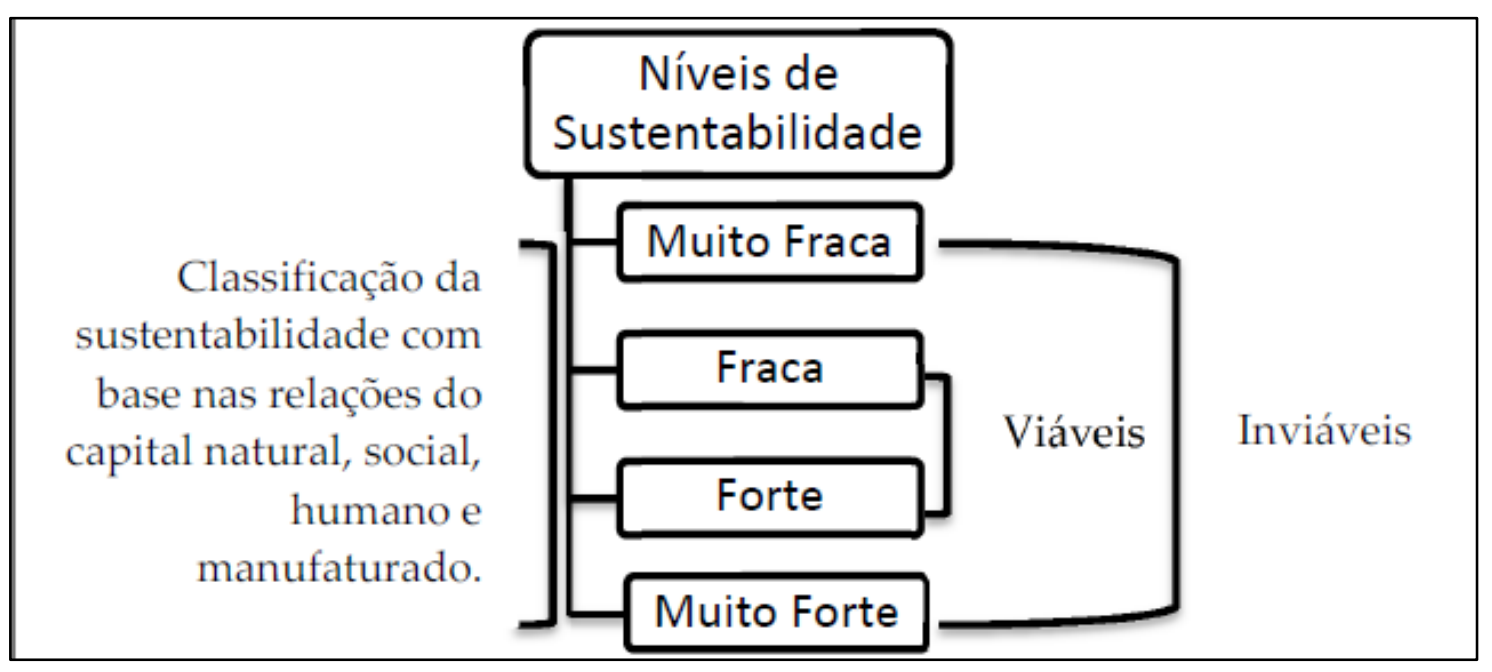

Figura 1. Classificação e características dos níveis de sustentabilidade.

Garmendia et al. (2010) salienta que, independente do nível no qual a sustentabilidade se enquadra, o bemestar da sociedade não deve diminuir ao longo do tempo. Porém, nota-se que essa afirmação é incoerente com as afirmações de Gaurav et al. (2011) e Howard et al. (2014) de que os humanos dependem totalmente dos recursos naturais para sua existência e bem-estar.

A escolha adequada de um nível de sustentabilidade compreende um meio termo entre a sustentabilidade fraca e a forte (Romero e Linares, 2010). Essa afirmação é considerada consistente, pois as sustentabilidades muito fraca e muito forte são inviáveis na questão do capital natural.

A análise deste nível de sustentabilidade gerou uma vasta preocupação sobre a forma de mensurá-la. Sendo assim, na sequência, apresentam-se as dimensões e sua sustentabilidade, consideradas antecessoras dos indica- dores de mensuração de sustentabilidade.

As dimensões e a sustentabilidade ambiental, social e econômica

A sustentabilidade abrange as dimensões ambientais, sociais e econômicas, e estas sofrem interações que ocorrem nas mais variadas formas (Krajnc e Glavic, 2003; Aktas et al., 2013). Além disso, a sustentabilidade abrange as dimensões, e dentro destas é desenvolvida cada uma das sustentabilidades específicas da TBL.

A dimensão ambiental compreende o (Labuschagne et al., 2005): a) recurso ar (qualidade, toxicidade, acidificação, aquecimento global, camada de ozônio, emissões atmosféricas); b) recurso hídrico (qualidade e quantidade, lançamento de efluentes e poluentes); c) recurso terrestre (qualidade e quantidade, poluentes do solo, uso da terra); e d) recurso mineral e energético 
(esgotamento de recursos energéticos e minerais não renováveis).

A sustentabilidade ambiental objetiva proteger as fontes de matériasprimas necessárias às inevitabilidades humanas e que os dejetos humanos não sejam excedidos de seus sumidouros, evitando danos aos humanos (Goodland, 1995). Portanto, centra-se na vitalidade e na saúde dos sistemas biogeofísicos, integrando-os à multiescala global, às dinâmicas, às hierarquias dos sistemas, à organização, entre outros. Essa afirmação também pode ser vista em Ciegis et al. (2009) e Moldan et al. (2012).

A sustentabilidade ambiental como a manutenção dos serviços da natureza dentro de um nível adequado, tendo em mente a indivisibilidade destes serviços e o bem-estar humano (Ekins et al., 2003). Sendo assim, esses serviços são fornecidos apenas quando as condições dos sistemas ecológicos globais estiverem em estado saudável e apresentarem biodiversidade biológica. Sartori et al. (2014) definem-na como a desmaterialização da atividade econômica, pois uma redução do consumo de capital natural reduz a pressão sobre os sistemas naturais e, assim, amplia a prestação de serviços ambientais à sociedade.

A dimensão ambiental reflete a área de abrangência, tais como os sistemas biogeofísicos, que são compostos pelo ar, pela água, pelo solo, pela energia, pelos recursos minerais, entre outros, a nível planetário. Essa dimensão abrange a sustentabilidade ambiental, a qual tem como propósito a proteção e manutenção da vitalidade dos sistemas biogeofísicos. Deve-se considerar que se trata de sistemas complexos, com capacidade dinâmica de adaptação. Portanto, a sustentabilidade ambiental vai além da manutenção de um status quo ideal e da perspectiva de proteção dos sistemas naturais para o estreito objetivo econômico. Essas reflexões corroboram Ciegis et al. (2009) e Moldan et al. (2012).
A dimensão social abrange (Labuschagne et al., 2005; Glavič e Lukman, 2007): a) os recursos humanos internos das empresas (estabilidade de emprego, práticas de emprego, saúde ocupacional e segurança, salários e benefícios, desenvolvimento de capacidades); b) a população externa da empresa (capital humano, capital produtivo, capital comunidade); c) a participação dos stakeholders (fornecimento de informações e influência dos stakeholders); e d) desempenho macrossocial (socioeconômico, socioambiental) Portanto, a dimensão social abrange todos os aspectos relacionados com bem-estar, política, cultura, instituições e outros, em nível local, nacional e global, coletivo e individual.

A sustentabilidade social centrase na preservação da capacidade de resistência, vigor e organização dos sistemas sociais e culturais. Sendo assim, entende-se que a sustentabilidade social compreende a continuação, no futuro, de valores, identidades, relações sociais e institucionais do presente. A sustentabilidade social ocorre quando as ações na sociedade ou nas instituições satisfizerem um vasto conjunto de necessidades, e é mister a preservação da reprodução do sistema natural em longo prazo e as reivindicações da justiça social, da dignidade humana e da participação social (Littig e Grießler, 2005).

Vallance et al. (2011) destacam que a sustentabilidade social possui três componentes: a) desenvolver: a satisfação das necessidades básicas, a equidade (inter)intrageração, a criação do capital social, a justiça, a liberdade, a infraestrutura e os serviços básicos, o emprego, a participação política generalizada, entre outros (McKenzie, 2004); b) interligar: a mudança comportamental no atendimento das metas ambientais biofísicas; e c) manter: os termos sociais, ou seja, preservar as características socioculturais frente a mudanças e as formas como a sociedade resiste a essas mudanças. Sendo assim, a 
sustentabilidade social refere-se à vitalidade da homogeneidade social, ao acesso a bens de serviço e ao emprego para todos, com remuneração justa, entre outros.

A dimensão econômica abrange a (ICHemE, 2002; Munasinghe, 2007): a) saúde financeira (lucratividade, liquidez e solvência); b) desempenho econômico (rentabilidade, contribuição com o PIB e participação de mercado); c) benefícios financeiros (incentivos à melhoria ambiental, social e ou tecnológica); e d) oportunidades comerciais (vulnerabilidade da rede de comércio e seus riscos). Sendo assim, entende-se que a dimensão econômica abrange as variáveis voltadas às questões econômicas e financeiras, incluindo os benefícios econômicos oferecidos pelas instituições à sociedade, em nível micro e macro.

A sustentabilidade econômica visa maximizar a geração do fluxo de renda e, ao mesmo tempo, manter o estoque de capital financeiro necessário para a geração desse rendimento (Azapagic, 2004). O GRI (2013) define-a como os impactos sobre o fluxo de renda de seus stakeholders e dos sistemas econômicos em âmbito local, nacional e global. Munasinghe (2007) destaca que a sustentabilidade econômica possui a responsabilidade de garantir produções e consumos ideais. Moldan et al. (2012) complementam que a utilização dos recursos atuais não pode limitar o fluxo de renda futuro.

Nesta lógica, salienta-se que a sustentabilidade econômica vincula-se à manutenção da geração de rendimento econômico e financeiro, em nível local, nacional e global, controlando a produção de produtos e serviços necessários para suprir a demanda hoje, sem comprometer a referida fabricação de bens diversos e a geração de renda no futuro. Sendo assim, a manutenção do capital natural é necessária para evitar o decréscimo econômico.

Em suma, a definição das dimensões da sustentabilidade compreende as extensões e/ou as áreas abrangidas, em qualquer sentido, e dividem-se, considerando o conceito TBL Figura 2. Essas três dimensões aderemse, essencialmente, às condições de vida hoje e seu melhoramento para o futuro.

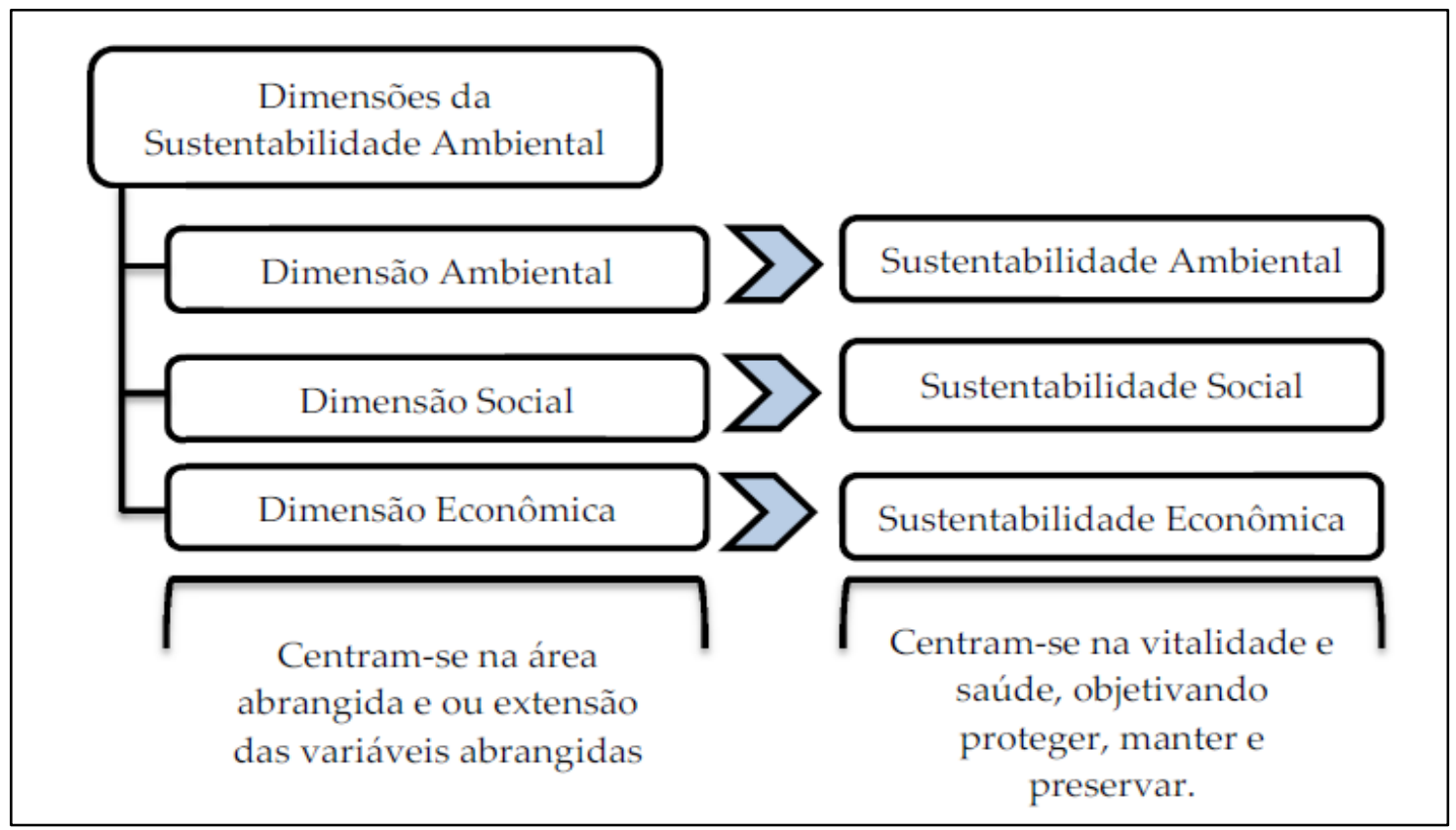

Figura 2. Estrutura das dimensões e da sustentabilidade ambiental, social e econômica. 
A sustentabilidade ambiental, social e econômica, dentro de cada uma das referidas dimensões (Figura 2), para ser mensurada, necessita de indicadores, e estes são apresentados e analisados na seção seguinte.

\section{dade}

\section{Indicadores de sustentabili-}

Os indicadores tradicionais e os indicadores de sustentabilidade estão representados na Figura 3. Além disso, nesta seção apresenta-se as críticas, as qualidades desejáveis e características específicas, a categoria dos indicadores e a forma de abordagem quanto a sua elaboração.

Os indicadores foram utilizados pioneiramente em contexto científico por
Lazarsfeld (1958). Os indicadores são variáveis que compreendem a qualidade, característica e a propriedade de um determinado sistema (Gallopin, 1997). Boulanger (2008) afirma que o indicador é uma variável observável, utilizada com a finalidade de expor a realidade não observável. Joung et al. (2012) definem o indicador como uma medida através da qual pode-se extrair uma conclusão sobre variáveis previamente definidas. Romero e Linares (2014) salientam que os indicadores normalmente mensuram uma dimensão na sua totalidade do cenário global da sustentabilidade, ou seja, abrangem todas as variáveis existentes para proporcionar uma visão global.

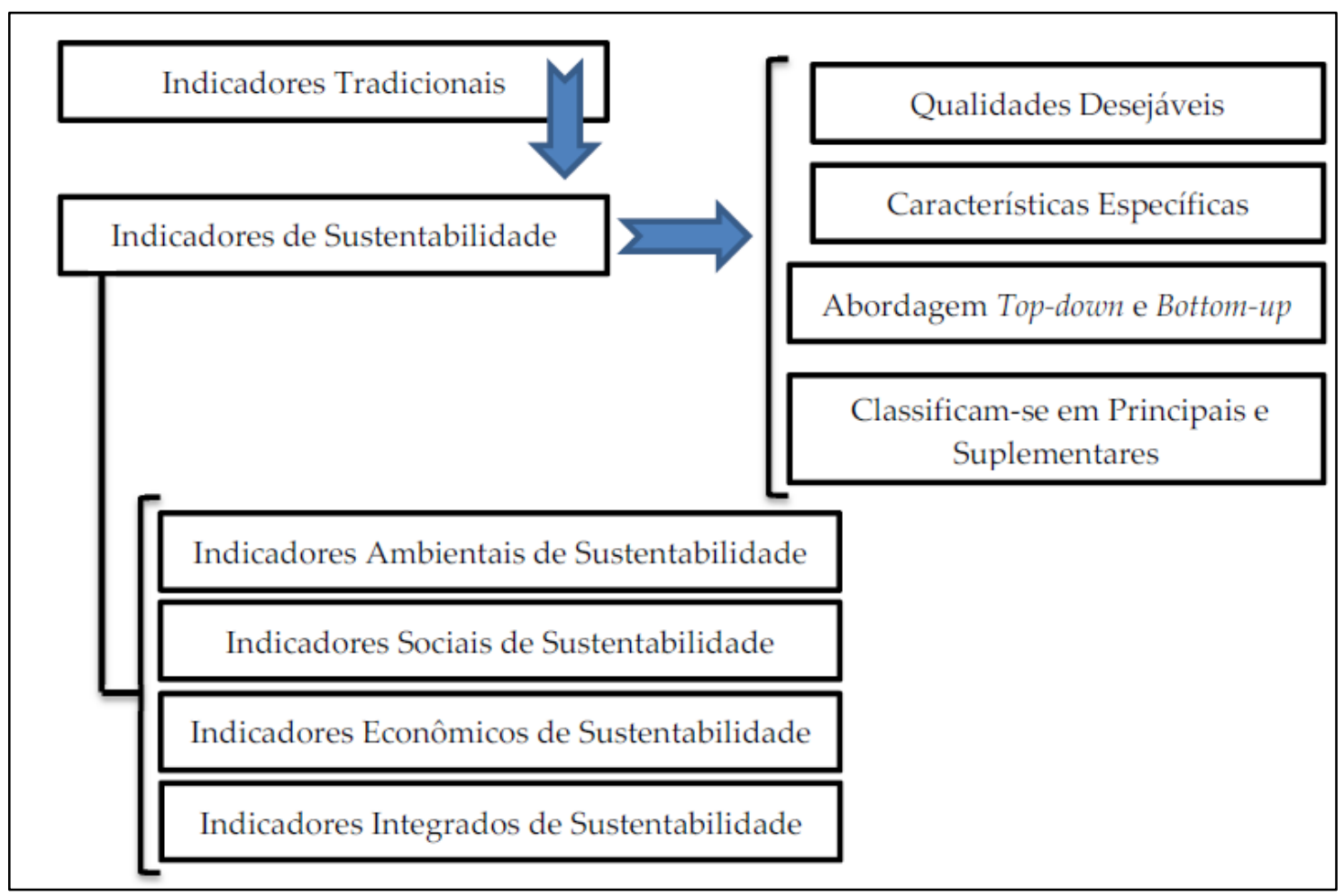

Figura 3. Indicadores tradicionais e de sustentabilidade.

A necessidade de indicadores exequíveis para orientar o processo da sustentabilidade foi reconhecida pela conferência Rio'92 e na Agenda 21 (UNCED, 1992). Portanto, uma série de indicadores de sustentabilidade, desde a 
Rio'92, foram elaborados para auxiliar os processos decisórios vinculados à sustentabilidade e difundidos em nível global, corroborando com Dahl (2012).

Os indicadores de sustentabilidade correspondem às informações confiáveis utilizadas na mensuração das metas e dos objetivos, bem como a motivação para o avanço em direção às metas, aos objetivos ou aos níveis de sustentabilidade (Romero e Linares, 2014). Esses indicadores devem quantificar os processos dos sistemas ambiental-humano, com equidade, para garantir a sua continuidade $e$ funcionalidade no futuro (Hak et al., 2007). Portanto, a denominação sistema ambiental-humano compreende apenas um único e indivisível sistema, que é o sistema global, e abrange todos os tipos de capitais; a equidade compreende a quantificação com igualdade dos sistemas envolvidos.

Os indicadores de sustentabilidade simplificam as informações (Singh et al., 2012), quantificam-nas (Lahtinen et al., 2014), analisam-nas (Hardi e Zdan, 2010; Singh et al., 2012), e comunicam as informações complexas de forma simples (Krajnc e Glavic, 2003; Hak et al., 2007). Sendo assim, para Hardi e Zdan (2010), os indicadores de sustentabilidade representam medidas sistemáticas, precisas, consistentes e transparentes das dimensões da sustentabilidade. Linke et al. (2013) enfatizam que os indicadores oferecem uma solução simples e acessível para a avaliação rápida da sustentabilidade.

Os indicadores de sustentabilidade agrupam processos complexos e diversificados em poucas medidas e de forma simples, maximizando a informação original e relevante (Agol et al., 2014). Porém, ressalta-se que esta pretensão, independente da quantidade de indicadores utilizados, não consegue traduzir todas as questões envolvidas; sendo assim, eles geram incertezas quanto às medidas e, desta forma, não representam a realidade, mas apenas se aproximam desta. Essa reflexão crítica também é feita por Gaurav et al. (2011) e Lodhia e Martin (2014).

Entende-se, também, que o indicador de sustentabilidade mais sofisticado pode não representar necessáriamente a escolha mais adequada, ou seja, um conjunto de indicadores, independente da quantidade, pode não ser completo. Sendo assim, deve-se entender que não é possível medir tudo dentro de um sistema e que algo sempre será perdido. Essa reflexão também pode ser vista em Searcy et al. (2007) e Chee Tahir e Darton (2010).

Além dessas críticas sobre os indicadores de sustentabilidade, estas são as principais existentes na literatura: a) inexistência de benchmark de referência (Munda, 2006); b) dificuldades na interpretação do indicador, principalmente quando há uma enorme agregação de informações (Hezri e Dovers, 2006); c) possuir informações explicativas revelando sintomas em vez de causas e, além disso, possuir inadequação da escala espacial (Bell e Morse, 2008); d) elaborados em função do que pode em vez do que deve ser medido (McCool, 2004); e e) indicadores simples são inapropriados para demonstrar toda a realidade de um sistema complexo adaptativo (Milman e Short, 2008).

Com base nessas críticas, entende-se que até o momento não existem metodologias mais adequadas para a mensuração da sustentabilidade. Dessa forma, apesar dos indicadores não representarem as opções mais adequadas da mensuração da sustentabilidade, ainda compreendem a forma mais plausível, pois é melhor medir algo de forma vaga do que não medir. Existem diversos motivos apresentados pela literatura corroborando essa informação, e eles são apresentados a seguir.

Os indicadores de sustentabilidade são dados utilizáveis nas tomadas de decisões para intervir no sistema e corrigi-lo em função dos objetivos propostos, ajudando assim a 
criar um cenário desejado do ambiente e da sociedade, que deve ser protegida e promovida (McCool, 2004; Hezri e Dovers, 2006; Lahtinen et al., 2014). Assim, os indicadores de sustentabilidade podem fornecer um quadro preliminar, estimulando a concepção de uma série de ações preventivas, soando um alarme com tempo hábil para evitar impactos ambientais, sociais e econômicos, estabelecer prioridades, formular estratégias e testar sua eficácia.

Os indicadores possuem 0 potencial para identificar se o objeto em estudo (empresa, instituição, nação) está próximo ou distante do padrão de sustentabilidade desejado (Azapagic e Perdan, 2000; Bohringer e Jochem, 2007; Milman e Short, 2008; Lodhia e Martin, 2014).

Os indicadores apontam múltiplas questões da sustentabilidade e auxiliam na determinação da situação do sistema atual e regressões futuras. Além disso, servem como sistema de monitoramento e precaução relacionados com o clima, o ambiente e a sociedade (Lahtinen et al., 2014; Lodhia e Martin, 2014). São também utilizados na realização de comparações no tempo e no espaço, ou com os objetivos propostos e a avaliação de desempenho (Milman e Short, 2008). Veleva et al. (2001) apontam três objetivos principais desses indicadores: a) aumentar a conscientização e compreensão da situação; b) abastecer de informação a tomada de decisão; e c) mensurar o avanço na direção dos objetivos propostos.

A literatura também apresenta qualidades desejáveis (Tabela 1), que devem ser observadas na elaboração e identificação dos indicadores de sustentabilidade.

Tabela 1. Qualidades desejáveis dos indicadores de sustentabilidade.

\begin{tabular}{|l|}
\hline \multicolumn{1}{|c|}{ Qualidades } \\
\hline $\begin{array}{l}\text { Terem base em informações confiáveis a partir de fontes de dados válidos, disponíveis, precisos e } \\
\text { acessíveis. }\end{array}$ \\
\hline Serem tecnicamente mensuráveis, reprodutíveis, com custo razoável e de fácil aplicação e avaliação. \\
\hline Elaborados e avaliados a partir de um processo aberto com participação dos stakeholders. \\
\hline Permitir comparações entre corporações, e avaliar seu desempenho sustentável no tempo. \\
\hline $\begin{array}{l}\text { Serem indicadores simples, mas significativos. Compreenderem um conjunto de indicadores } \\
\text { gerenciável, em vez de um único que em sua composição contenha indicadores principais e } \\
\text { específicos. }\end{array}$ \\
\hline Devem compreender aspectos qualitativos e quantitativos, mensuráveis na forma quantitativa. \\
\hline $\begin{array}{l}\text { Abordarem questões globais e uma visão holística: do berço à morte, o conteúdo deve ser claro, } \\
\text { compreensível e transparente. }\end{array}$ \\
\hline Devem ser maleáveis no tempo e terem apropriada escala temporal e espacial. \\
\hline
\end{tabular}

Fonte: Adaptado de Bossel (1999), Veleva e Ellenbecker (2001), Searcy et al. (2007), Azapagic (2004), Patlitzianas et al. (2008), Nordheim e Barrasso (2007), Gaurav et al. (2011).

Os indicadores de sustentabilidade, além das qualidades desejáveis, também devem apresentar especificações, elencadas a partir dos estudos e que compreendem (Krajn e Glavic, 2003; Patlitzianas et al., 2008): a) o período de acompanhamento e cálculo (ano, trimestre, bimestre ou mensal); b) o limite, ou seja, o nível de abrangência na organização hierárquica (linha de produtos, instalações, ciclo de vida do produto e/ou fornecedores); c) a unidade de medida (números, quilogramas, toneladas, dólares, porcen- 
tagem e horas); d) o tipo de medição: ajustado (energia por unidade de produto ou serviço por ano) ou absoluto (total de energia por ano em $\mathrm{KWh}$ ), qualitativo ou quantitativo; e Patlitzianas et al. (2008) complementa com e) a identificação alfanumérica único do indicador; f) sua denominação, contendo sua designação distintiva; g) a definição das características essenciais e a função; e h) serem fundamentados e referenciados em base teórica ou prédesenvolvidos, adequação técnica e científica.

Após revisar as qualidades desejáveis e as características dos indicadores de sustentabilidade, necessita-se revisar sua forma de elaboração e identificação. Porém, ressalta-se que os indicadores desenvolvidos para avaliação de situações específicas, em geral, não são adequados na utilização em todos os contextos das medições. Sendo assim, devem-se exigir modificações para se adequar a outras ou novas situações na mensuração da sustentabilidade, também corroborado por Joung et al. (2012).

A elaboração de indicadores e suas modificações pode ocorrer pela abordagem top-down e bottom-up (Lahtinen et al., 2014). Os indicadores elaborados utilizando a top-down advêm de processos políticos selecionados a partir do conhecimento de especialistas e pesquisadores e são aplicados nas tomadas de decisões em nível macro; são, assim, projetados para aplicação em grandes organizações e/ou áreas geográficas (Chee Tahir e Darton, 2010). Analisando-se a abordagem top-down, percebe-se que sua fragilidade compreende a perda de questões críticas em nível local, ou seja, comunidades locais.

Os indicadores com abordagem bottom-up são elaborados com a participação sistemática de diversos stakeholders locais, ou seja, em nível micro, considerando as adversidades locais (Chee Tahir e Darton, 2010). A abordagem bottom-up enfatiza a importância de abranger o contexto local e suas necessidades especiais, o que permite que as comunidades locais participem da seleção, coleta e do monitoramento dos indicadores de sustentabilidade (Reed et al., 2006).

Desta forma, as tendências atuais na determinação de indicadores de sustentabilidade ocorrem de forma híbrida, observando as abordagens topdown e a bottom-up, nas quais os indicadores são formados por especialistas e pesquisadores, mas as escolhas realizadas por eles dependem das preferências políticas e sociais da comunidade local. Essa integração aumenta a operacionalização e política dos indicadores, também apoiado por Reed et al. (2006) e Turcu (2013).

Os indicadores de sustentabilidade, segundo Manara e Zabaniotou (2014), possuem categorias e englobam: a) os indicadores sociais de sustentabilidade; b) os indicadores ambientais de sustentabilidade; c) os indicadores econômicos de sustentabilidade; e d) os indicadores integrados de sustentabilidade.

Os indicadores de sustentabilidade ambiental devem refletir a sucessão de ocorrências que conduzem a um determinado efeito resultante, onde cada membro da sucessão causa a ocorrência do efeito seguinte, vinculando a atividade humana aos seus impactos ambientais (Smeets e Weterings, 1999). Estes indicadores devem considerar a abordagem do ciclo de vida e a abordagem completa das interações humanas com o meio ambiente, identificando os hotspots (prioridade de conservação) que podem ser direcionados a melhorias (Azapagic e Perdan, 2000). Portanto, os indicadores ambientais de sustentabilidade medem os impactos locais, regionais ou globais sobre os sistemas naturais, que abrangem seres humanos, ecossistemas, terra, ar e água, e que afetam um grande número de interessados.

Os indicadores sociais de sustentabilidade, segundo Azapagic 
(2004) e Manara e Zabaniotou (2014), devem considerar os vários interesses dos funcionários e das comunidades, revelando os impactos sociais em nível local, nacional e global. Carrera e Mack (2010) salientam que esses indicadores vinculam-se à avaliação de impacto social, que consiste em uma metodologia para monitorar e analisar as consequências não intencionais das intervenções previstas, como a implementação de novas tecnologias.

Percebe-se que os indicadores sociais de sustentabilidade pretendem obter uma visão holística, incluindo diversas dimensões. Sendo assim, a mensuração dos impactos sociais inclui os indicadores sobre a saúde, os riscos e os impactos educacionais. Esses indicadores são aplicados no contexto das perturbações naturais e antropogênicas. Dessa forma, medem a resposta do ecossistema frente a perturbações antrópicas.

Os indicadores econômicos da sustentabilidade medem o impacto econômico frente aos stakeholders endógenos e exógenos, assim como os ambientais e sociais, em nível local, nacional e global, abrangendo as medidas usuais de desempenho financeiro (lucros e retornos), além de medidas mais amplas (Manara e Zabaniotou, 2014).

Os indicadores integrados de sustentabilidade compreendem a interrelação de dois ou mais indicadores de diferentes dimensões em uma medida de desempenho (Azapagic, 2003; 2004). Salientam ainda que esta integração, por um lado, reduz o número de indicadores, traz maior gerenciamento das medidas e facilita o processo de tomada de decisão. Por outro lado, considerando que a sustentabilidade é um conceito holístico, deve haver um esforço para considerar simultaneamente as três dimensões da sustentabilidade. Os indicadores integrados aproximam $\mathrm{o}$ atingimento deste objetivo (Lozano e Huisingh, 2011).

Portanto, os indicadores de sustentabilidade devem abranger a integração das três dimensões (ambiental, social e econômica), permitindo assim ter uma visão holística da sustentabilidade, além dos indicadores específicos de cada dimensão, o que corrobora com Lozano e Huisingh (2011) e Lodhia e Martin (2014).

Os indicadores ambientais, sociais e econômicos de sustentabilidade classificam-se em principais e suplementares (Veleva e Ellenbecker, 2001). Estes autores ainda enfatizam que os indicadores principais compreendem um conjunto padrão de indicadores e podem ser aplicados a qualquer empresa e/ou instituição. Esse conjunto de indicadores principais é bem difundido, tais como o quadro de indicadores do Global Reporting Initiative (GRI) (GRI, 2013), o Commission on Sustainable Development (CSD) (CSD, 2007), do Institution of Chemical Engineers (ICHEME, 2002), Wuppertal Institute for Climate, Environment, Energy (Spangenberg e Bonniot, 1998), a International Organization for Standardization (ISO) número 14031:2013 (ISO, 2013), e o Instituto Ethos (ETHOS, 2016)

Os indicadores suplementares são compreendidos por um conjunto aberto e podem variar entre empresas e/ou instituições. Sendo assim, introduzem alguma flexibilidade, abordando aspectos específicos (Veleva e Ellenbecker, 2001). Esses indicadores suplementares são amplamente disponíveis e encontrados, por exemplo, nos estudos de Nordheim e Barrasso (2007) e Searcy et al. (2007).

Sobre os indicadores principais, afirma-se que não podem ser considerados melhores ou mais importantes que os indicadores suplementares. O ideal é a utilização mista: tanto os principais quanto os suplementares devem fazer parte dos indicadores. Cabe salientar que os indicadores principais são os resultantes da abordagem top-down e os suplementares da abordagem bottom-up. 


\section{Conclusões}

Recomenda-se que a operacionalização de qualquer uma das dimensões da sustentabilidade ocorra com base em indicadores; no entanto, percebe-se, que os indicadores tradicionais ficam aquém de cobrir as questões da sustentabilidade. Dessa forma, necessita-se de indicadores específicos para medir a sustentabilidade. Portanto, este estudo possui como objetivo principal analisar os níveis, as dimensões e os indicadores de sustentabilidade, para melhorar 0 entendimento e a clareza destas questões essenciais na mensuração dela.

A análise da classificação dos níveis de sustentabilidade em muito fraca, fraca, forte e muito forte revela que se vinculam à substituição do capital natural ou não por outros capitais existentes. Percebe-se que a sustentabilidade muito forte e muito fraca são inviáveis frente ao capital natural; já os níveis forte e fraca são viáveis, mas a escolha de um meio termo entre os dois é a opção mais adequada. A sustentabilidade abrange dimensões, e a análise dessas dimensões revela que a dimensão ambiental, social e econômica vincula-se à identificação da extensão e/ou da área abrangida de cada uma das variáveis. Essas dimensões dividem-se em sustentabilidade ambiental, social e econômica e estas últimas centram-se na vitalidade e ou na saúde de cada um dos sistemas com o objetivo de manter, proteger e preservar.

A sustentabilidade ambiental, social e econômica necessita de indicadores para ser mensurada. Sendo assim, os indicadores de sustentabilidade abrangem os indicadores ambientais, sociais e econômicos de sustentabilidade. A análise dos indicadores de sustentabilidade revelou que, para sua elaboração e identificação, deve-se observar as qualidades desejáveis dos indicadores, assim como as suas características e especificações. As abordagens top-down e bottom-up na elaboração de indicadores de sustentabilidade devem ser utilizadas de forma híbrida, bem como os indicadores principais e suplementares.

Portanto, salienta-se que a definição desses termos da sustentabilidade, tais como os níveis, as dimensões e os indicadores, é escassa e muito dispersa na literatura. Dessa forma, este estudo buscou agregar essas informações, procurando contribuir com demais estudos centrados neste tema.

\section{Conflitos de interesse}

Os autores declaram não haver conflitos de interesse.

\section{Referências}

Agol, D.; Latawiec, A. E.; Strassburg, B. Evaluating impacts of development and conservation projects using sustainability indicators: Opportunities and challenges. Environmental Impact Assessment Review, v 48, p. 1-9, 2014. https://doi.org/10.1016/j.eiar.2014.04.001

Aktas, R.; Kayalidere, K.; Kargin, M. Corporate sustainability reporting and analysis of sustainability reports in Turkey. International Journal of Economics and Finance, v. 5, n. 3, p. 113-125, 2013. https://doi.org/10.5539/ijef.v5n3p113

Ayres, R. U. On the practical limits to substitution. Ecological Economics, v. 61, n. 1, p. 115-128, 2007. https://doi.org/ 10.1016/j.ecolecon.2006.02.011

Azapagic, A.; Perdan, S. Indicators of sustainable development for industry: a general framework. Process Safety and Environmental Protection, v. 78, n. 4, p. 243-261, 2000. https://doi.org/10.1205/095 758200530763

Azapagic, A. Systems approach to corporate sustainability: A general management framework. Process Safety and Environmental Protection, v. 81, p. 303316, 2003. https://doi.org/10.1205/ 095758203770224342

Azapagic, A. Developing a framework for sustainable development indicators for the mining and minerals industry. Journal of 
Cleaner Production, v. 12, p. 639-662, 2004. https://doi.org/10.1016/S0959-6526(03) 00075-1

Bell, S.; Morse, S. Sustainability indicators: Measuring the immeasurable? London: Earthscan, 2008.

Blewitt, J. Understanding sustainable development. London: Earthscan, 2008.

Bohringer, C.; Jochem, P. Measuring the immeasurable: A survey of sustainability indices. Ecological Economics, v. 63, p. 1-8, 2007. https://doi.org/10.1016/j.ecolecon. 2007.03.008

Boulanger, P. Sustainable development indicators: A scientific challenge, a democratic issue. Surveys and Perspectives Integrating Environment and Society, v. 1, p. $59-73,2008$.

Bossel, $H$. Indicators for sustainable development: Theory, method, applications: A report to the Balaton Group. Winnipeg: International Institute for Sustainable Development (IISD), 1999.

Carrera, D. G.; Mack, A. Sustainability assessment of energy technologies via social indicators: Results of a survey among European energy experts. Energy Policy, v. $38, \quad$ n. $2, \quad$ p. 1030-1039, 2010. https://doi.org/10.1016/j.enpol.2009.10.055

Chee Tahir, A.; Darton, R. C. The process analysis method of selecting indicators to quantify the sustainability performance of a business operation. Journal of cleaner production, v. 18, p. 1598-1607, 2010. https://doi.org/10.1016/j.jclepro.2010.07.012

Ciegis, R.; Ramanauskiene, J.; Martinkus, B. The concept of sustainable development and its use for sustainability scenarios. Engineering Economics, v. 2, n. 62, p. 28-37, 2009.

CSD - Commission on Sustainable Development. Indicators of sustainable development: Guidelines and methodologies. 3. ed. Washington: United Nations Publication, 2007.

Dahl, A. L. Achievements and gaps in indicators for sustainability. Ecological Indicators, $\quad$ v. 17, p. 14-19, 2012. https://doi.org/10.1016/j.ecolind.2011.04.0 32
Daly, H. E. Steady-state economics with new essays. 2. ed. London: Earthscan, 1992.

Ekins, P.; Simon, S.; Deutsch, L.; Folke, C.; Groot, R. A Framework for the practical application of the concepts of critical natural capital and strong sustainability. Ecological Economics, v. 44, p. 165-185, 2003. https://doi.org/10.1016/S0921-8009 (02)00272-0

ETHOS - Instituto Ethos de Empresas e Responsabilidade Social. Indicadores Ethos para negócios sustentáveis e responsáveis. $2016 . \quad$ Disponível em: <http://www3.ethos.org.br/conteudo/iniciat ivas/indicadores/>. Acesso em: 12 abr. 2019.

Fiorino, D. J. Explaining national environmental performance: Approaches, evidence, and implications. Policy Sciences, v. 44, n. 4, p. 367-389, 2011. https://doi.org/ $10.1007 / \mathrm{s} 11077-011-9140-8$

Garmendia, E.; Prellezo, R.; Murillas, A.; Escapa, M.; Gallastegui, M. Weak and strong sustainability assessment in fisheries. Ecological Economics, v. 70, n. 1, p. 96-106, 2010. https://doi.org/10.1016/j.ecolecon. 2010.08.001

Gallopin, G. Indicators and their use: Information for decision-making. In: Moldan, B.; Billharz, S. Sustainability indicators: Report of the project on indicators of sustainable development. Nova Jersey: John Wiley and Sons, 1997.

Gaurav, A.; Rachuri, S.; Fiorentini, X.; Mani, M.; Fenves, S. J.; Lyons, K. W.; Sriram, R. D. Extending the notion of quality from physical metrology to information and sustainability. Journal of Intelligent Manufacturing, v. 22, n. 5, p. 737-750, 2011. https://doi.org/ 10.1007/s10845-009-0333-3

Glavič, P.; Lukman, R. Review of sustainability terms and their definitions. Journal of Cleaner Production, v. 15 , n. 18 , p. 18751885, 2007. https://doi.org/10.1016/ j.jclepro.2006.12.006

Goodland, R. The concept of environmental sustainability. Annual Review of Ecology and Systematics, v. 26, p. 1-24, 1995. https://doi.org/10.1146/annurev.es.26.1101 95.000245 
Goodland, R.; Daly, H. Environmental sustainability: Universal and non-negotiable. Ecological Applications, v. 6, n. 4, p. 10021017, 1996. https://doi.org/10.2307/ 2269583

GRI - Global Reporting Initiative. Reporting guidelines (the guidelines) offer reporting principles: Standard disclosures. 2013. Disponível em: <https://www.global reporting.org/resourcelibrary/grig4-part1reporting-principles-and-standarddisclosures.pdf>. Acesso em: 12 abr. 2019.

Hak, T.; Moldan, B.; Dahl, A. L. Sustainability indicators: A scientific assessment. Washington: Island Press, 2007.

Hardi, P.; Zdan, T. Assessing sustainable development: Principles in practice. Winnipeg: International Institute for Sustainable Development (IISD), 2010.

Hezri, A. A.; Dovers, S. R. Sustainability indicators, policy and governance: Issues for ecological economics. Ecological Economics, v. 60 , n. 1, p. 86-99, 2006. https://doi.org/ 10.1016/j.ecolecon.2005.11.019

Horbach, J. Indicator systems for sustainable innovation. Heidelberg: Physica-Verlag, 2005. https://doi.org/ $10.1007 / \mathrm{b} 138695$

Howard, G. R.; Lubbe, S.; Huisman, M.; Klopper, R. Green IS management framework verification: Explicating the enabling capabilities of green IS. Proceedings of the 28th Enviro Info. Conference, Oldenburg, Germany, 2014.

ICHEME - Institution of Chemical Engineers. The sustainability metrics: Sustainable development progress metrics recommended for use in the process industries. Rugby: Institution of Chemical Engineers, 2002. Disponível em: <http://nbis.org/ nbisresources/metrics/triple_bottom_line_in dicators_process_industries.pdf $>$. Acesso em: 12 abr. 2019.

ISO - International Organization For Standardization. ISO 14031:2013 (en) Environmental management Environmental performance evaluation Guidelines. 2013. Disponível em: <https://www.iso.org/obp/ui/\#iso:std:iso:1 4031:ed-2:v1:en>. Acesso em: 12 abr. 2019.

Joung, C. B.; Carrell, J.; Sarkar, P.; Feng, S. C. Categorization of indicators for sustainable manufacturing. Ecological indicators, v. 24, p. 148-157, 2012. https://doi.org/10.1016/ j.ecolind.2012.05.030

Labuschagne, C.; Brent, A. C.; Erck, R. P. Assessing the sustainability performances of industries. Journal of Cleaner Production, v. 13, n. 4, p. 373-385, 2005. https://doi.org/ 10.1016/j.jclepro.2003.10.007

Lahtinen, K.; Myllyviita, T.; Leskinen, P.; Pitkänen, S. K. A systematic literature review on indicators to assess local sustainability of forest energy production. Renewable and Sustainable Energy Reviews, v. 40, p. 12021216, 2014. https://doi.org/10.1016/ j.rser.2014.07.060

Lazarsfeld, P. Evidence and inference in social research. Daedalus, v. 87, n. 4, p. 99109, 1958.

Linke, B. S.; Corman, G. J.; Dornfeld, D. A.; Tönissen, S. Sustainability indicators for discrete manufacturing processes applied to grinding technology. Journal of Manufacturing Systems, v. 32, n. 4, p. 556563, 2013. https://doi.org/10.1016/j.jmsy. 2013.05.005

Littig, B.; Grießler, E. Social sustainability: A catchword between political pragmatism and social theory. International Journal for Sustainable Development, v. 8, n. 1/2, p. 65-79, 2005.

Lodhia, S.; Martin, N. Corporate sustainability indicators: An Australian mining case study. Journal of Cleaner Production, v. 84, p. 107-115, 2014. https://doi.org/10.1016/ j.jclepro.2014.05.050

Lozano, R.; Huisingh, D. Inter-linking issues and dimensions in sustainability reporting. Journal of Cleaner Production, v. 19, p. 99107, 2011. https://doi.org/10.1016/j.jclepro. 2010.01.004

Krajnc, D.; Glavic, P. Indicators of sustainable production. Clean Technologies and Environmental Policy, v. 5, p. 279-288, 2003. https://doi.org/10.1007/s10098-0030221-z

Manara, P.; Zabaniotou, A. Indicator-based economic, environmental, and social sustainability assessment of a small gasification bioenergy system fuelled with food processing residues from the Mediterranean agro-industrial sector. Sustainable Energy Technologies and 
Assessments, v. 8, p. 159-171, 2014. https://doi.org/10.1016/j.seta.2014.08.007

McCool, S. F. Indicators of sustainability: Challenges and opportunities at the interface of science and policy. Environmental Management, v. 33, n. 3, p. 294-305, 2008. https://doi.org/10.1007/s00267-003-00844

McKenzie, S. Social sustainability: Towards some definitions. Adelaide: Hawke Research Institute, University of South Australia, 2004.

Milman, A.; Short, A. Incorporating resilience into sustainability indicators: An example for the urban water sector. Global Environmental Change, v. 18, p. 758-767, 2008. https://doi.org/10.1016/j.gloenvcha. 2008.08.002

Moldan, B.; Janoušková, S.; Hák, T. How to understand and measure environmental sustainability: Indicators and targets. Ecological Indicators, v. 17, p. 4-13, 2012. https://doi.org/10.1016/j.ecolind.2011.04.0 33

Munasinghe, M. Sustainable development triangle. Encyclopedia of Earth (EoE), 2007.

Munda, G. A NAIADE based approach for sustainability benchmarking. International Journal of Environmental Technology and Management, v. 6, n. 1/2, p. 65-78, 2006.

Neumayer, E. Weak versus strong sustainability: Exploring the limits of two opposing paradigms. Cheltenham: Edward Elgar, 2003.

Nordheim, E.; Barrasso, G. Sustainable development indicators of the European aluminium industry. Journal of Cleaner Production, v. 15, n. 3, p. 275-279, 2007. https://doi.org/10.1016/j.jclepro.2006.02.004

Pisani, J. A. Sustainable development: Historical roots of the concept. Environmental Sciences, v. 3, n. 2, p. 83-96, 2006. https://doi.org/10.1080/1569343060 0688831

Patlitzianas, K. D.; Doukas, H.; Kagiannas, A. G.; Psarras, J. Sustainable energy policy indicators: Review and recommendations. Renew Energy, v. 33, n. 5, p. 966-973, 2008. https://doi.org/10.1016/j.renene.2007.05.00 3
Reed, M. S.; Fraser, E. D. G.; Dougill, A. J. An adaptive learning process for developing and applying sustainability indicators with local communities. Ecological Economics, v. 59, p. 406-418, 2006. https://doi.org/10.1016/ j.ecolecon.2005.11.008

Rocha, C. M. R. S.; Gadelha, D. P.; Almeida, S. R. $\mathrm{O}$ desenvolvimento de políticas públicas e a influência do capital social na construção do desenvolvimento local sustentável. Revista Brasileira de Gestão Ambiental e Sustentabilidade, v. 4, n. 8, p. 463-474, 2017 https://doi.org/10.21438/rbgas. 040818

Romero, J. C.; Linares, P. Exergy as a global energy sustainability indicator. A review of the state of the art. Renewable and Sustainable Energy Reviews, v. 33, p. 427442, 2014. https://doi.org/10.1016/j.rser. 2014.02.012

Sartori, S.; Latrônico, F.; Campos, L. Sustainability and sustainable development: A taxonomy in the field of literature. Ambiente \& Sociedade, v. 17, n. 1, p. 1-20, 2014. Disponível em: <http://www.scielo.br/ pdf/asoc/v17n1/en_v17n1a02.pdf>. Acesso em: 12 abr. 2019.

Searcy, C.; McCartney, D.; Karapetrovic, S. Sustainable development indicators for the transmission system of an electric utility. Corporate Social Responsibility and Environmental Management, v. 14, n. 3, p. 135-151, 2007. https://doi.org/10.1002/ csr. 124

Singh, R. K.; Murty, H. R.; Gupta, S. K.; Dikshit, A. K. An overview of sustainability assessment methodologies. Ecological Indicators, $\quad$ v. 15, p. 281-290, 2012. https://doi.org/10.1016/j.ecolind.2011.01.007

Smeets, E.; Weterings, R. Environmental indicators: Typology and overview. Copenhagen: European Environmental Agency, 1999. (Technical Report, v. 25).

Solow, R. M. Intergenerational equity and exhaustible resources. Review of Economic $\begin{array}{llll}\text { Studies, } & \text { v. } 41, \quad \text { p. 29-46, } 1974 .\end{array}$ https://doi.org/10.2307/2296370

Spangenberg, J. H.; Bonniot, O. Sustainability indicators da compass on the road towards 
sustainability. Wuppertal Paper, n. 81, p.1-35, 1998. Disponível em: <https://www.econstor.eu/bitstream/10419 /49138/1/250130211.pdf>. Acesso em: 12 abr. 2019.

Turcu, C. Re-thinking sustainability indicators: local perspectives of urban sustainability. Journal of Environmental Planning and Management, v. 56, n. 5, p. $695-719,2013$. https://doi.org/10.1080/ 09640568.2012 .698984

Turner, R. K. Sustainability: Principles and practice. In: Turner, R. K. Sustainable Environmental Economics and Management: Principles and practice. New York: Belhaven Press, 1993. p. 3-36.

UNCED - United Nations Conference on Environment and Development. Agenda 21. Rio de Janeiro: UNCED, 1992.

Vallance, S.; Perkins, H. C.; Dixon, J. E. What is social sustainability? A clarification of concepts. Geoforum, v. 42, n. 3, p. 342-348, 2011. https://doi.org/10.1016/j.geoforum. 2011.01.002

Veleva, V.; Hart, M.; Greiner, T.; Crumbley, C. Indicators of sustainable production. Journal of Cleaner Production, v. 9, p. 447-452, 2001. https://doi.org/10.1016/S0959-6526 (01)00004-X

Veleva, V.; Ellenbecker, M. Indicators of sustainable production: A new tool for promoting business sustainability. NEW SOLUTIONS: A Journal of Environmental and Occupational Health Policy, v. 11, n. 1, p. 41-62, 2001. https://doi.org/10.2190/ XQK7-UB3W-3AQE-G4N0
WCED - World Commission on Environment and Development. Our common future. Oxford: Oxford University Press, 1987. Disponível em: <https://sswm.info/sites/ default/files/reference_attachments/UN\%20 WCED\%201987\%20Brundtland\%20Report. pdf>. Acesso em: 12 abr. 2019.

Wickboldt, L. A.; Moreira, J. A. P.; Silva, M. R.; Araújo, J. S.; Silva, J. V.; Pereira, J. A. Responsabilidade ambiental ou greenwash: uma análise da evidenciação ambiental das maiores empresas brasileiras. Revista Brasileira de Gestão Ambiental e Sustentabilidade, v. 5, n. 11, p. 897-910, 2018 . https://doi.org/10.21438/rbgas. 